\title{
Impact of an Oral Antineoplastic Renewal Clinic on Medication Possession Ratio and Cost-Savings
}

\author{
Brooke S. Crawford, PharmD, BCOP; Alison L. Stauder, PharmD, BCOP; Susan M. Bullington, PharmD, BCOP; \\ Patrick J. Kiel, PharmD, BCPS, BCOP, FHOPA; Erin R. Dark; Jill M. Johnson, PharmD, BCOP; \\ and Alan J. Zillich, PharmD, FCCP
}

\begin{abstract}
Purpose: The primary objective of this study was to evaluate the impact of a pharmacist-driven oral antineoplastic (OAN) renewal clinic on medication adherence and cost savings.

Methods: This was a preimplementation and postimplementation retrospective cohort evaluation within a single US Department of Veterans Affairs health care system following implementation of a pharmacist-managed OAN refill clinic. The primary outcome was medication adherence defined as the median medication possession ratio (MPR) before and after implementation of the clinic. Secondary outcomes included the proportion of patients who were adherent from pre- to postimplementation and estimated cost-savings of this clinic. Patients were eligible for inclusion if they had received at least 2 prescriptions of the most commonly prescribed oral antineoplastic agents at the institution between
\end{abstract}

September 1, 2013 and January 31, 2015.

Results: Of preimplementation patients, 96 of 99 (96.9\%) were male and all patients $(n=35)$ in the postimplementation group were male. The mean age of the preimplementation group was 69.2 years while the postimplementation group was 68.4 years. Median MPR in the preimplementation group was 0.94 , compared with 1.06 in the postimplementation group $(P<.001)$. Thirty-six (36.7\%) patients in the preimplementation group were considered nonadherent to their OAN regimen compared with zero patients in the postimplementation group. Estimated total cost savings was $\$ 36,335$ in the postimplementation period.

Conclusions: Implementation of a pharmacist-driven OAN renewal clinic was associated with a $12 \%$ increase in median MPR while saving an estimated $\$ 36,335$ during the 5-month postimplementation period.
Author affiliations can be found at the end of the article.

Correspondence:

Brooke Crawford

(brooke.crawford@va.gov)

Fed Pract. 2021;38. Published online March 22. doi:10.12788/fp.0107
5 valuation of oral antineoplastic agent (OAN) adherence patterns have identified correlations between nonadherence or over-adherence and poorer disease-related outcomes. Multiple studies have focused on imatinib use in chronic myeloid leukemia (CML) due to its continuous, long-term use. A study by Ganesan and colleagues found that nonadherence to imatinib showed a significant decrease in 5-year event-free survival between $76.7 \%$ of adherent participants compared with $59.8 \%$ of nonadherent participants. ${ }^{1}$ This study found that $44 \%$ of patients who were adherent to imatinib achieved complete cytogenetic response vs only $26 \%$ of patients who were nonadherent. In another study of imatinib for CML, major molecular response (MMR) was strongly correlated with adherence and no patients with adherence $<80 \%$ were able to achieve MMR. ${ }^{2}$ Similarly, in studies of tamoxifen for breast cancer, $<80 \%$ adherence resulted in a $10 \%$ decrease in survival when compared to those who were more adherent. ${ }^{3,4}$

In addition to the clinical implications of nonadherence, there can be a significant cost associated with suboptimal use of these medications. The price of a single dose of OAN medication may cost as much as $\$ 440$. $^{5}$
The benefits of multidisciplinary care teams have been identified in many studies. ${ }^{6,7}$ While studies are limited in oncology, pharmacists provide vital contributions to the oncology multidisciplinary team when managing OANs as these health care professionals have expert knowledge of the medications, potential adverse events (AEs), and necessary monitoring parameters. ${ }^{8}$ In one study, patients seen by the pharmacist-led oral chemotherapy management program experienced improved clinical outcomes and response to therapy when compared with preintervention patients (early molecular response, $88.9 \%$ vs $54.8 \%, P=.01$; major molecular response, $83.3 \%$ vs $57.6 \%, P=.06) .{ }^{9}$ During the study, 318 AEs were reported, leading to 235 pharmacist interventions to ameliorate AEs and improve adherence.

The primary objective of this study was to measure the impact of a pharmacistdriven OAN renewal clinic on medication adherence. The secondary objective was to estimate cost-savings of this new service.

\section{METHODS}

Prior to July 2014, several limitations were identified related to OAN prescribing and monitoring at the Richard L. Roudebush 
Veterans Affairs Medical Center in Indianapolis, Indiana (RLRVAMC). The prescription ordering process relied primarily on the patient to initiate refills, rather than the prescriber OAN prescriptions also lacked consistency for number of refills or quantities dispensed. Furthermore, ordering of antineoplastic products was not limited to hematology/oncology providers. Patients were identified with significant supply on hand at the time of medication discontinuation, creating concerns for medication waste, tolerability, and nonadherence.

As a result, opportunities were identified to improve the prescribing process, recommended monitoring, toxicity and tolerability evaluation, medication reconciliation, and medication adherence. In July of 2014, the RLRVAMC adopted a new chemotherapy order entry system capable of restricting prescriptions to hematology/oncology providers and limiting dispensed quantities and refill amounts. A comprehensive pharmacist driven OAN renewal clinic was implemented on September 1, 2014 with the goal of improving long-term adherence and tolerability, in addition to minimizing medication waste.

Patients were eligible for enrollment in the clinic if they had a cancer diagnosis and were concomitantly prescribed an OAN outlined in Table 1. All eligible patients were automatically enrolled in the clinic when they were deemed stable on their OAN by a hematology/oncology pharmacy specialist. Stability was defined as $\leq$ Grade 1 symptoms associated with the toxicities of OAN therapy managed with or without intervention as defined by the Common Terminology Criteria for Adverse Events (CTCAE) version 4.03. Once enrolled in the renewal clinic, patients were called by an oncology pharmacy resident (PGY2) 1 week prior to any OAN refill due date. Patients were asked a series of 5 adherence and tolerability questions (Table 2) to evaluate renewal criteria for approval or need for further evaluation. These questions were developed based on targeted information and published reports on monitoring adherence. ${ }^{10,11}$ Criteria for renewal included: $<10 \%$ self-reported missed doses of the OAN during the previous dispensing period, no hospitalizations or emergency department visits since most recent hematology/oncology provider appointment,
TABLE 1 Eligible Antineoplastic Agents for Enrollment in the Renewal Clinic

\begin{tabular}{ll} 
Diagnosis & Agents \\
\hline Chronic myeloid leukemia & Dasatinib, imatinib, nilotinib \\
\hline Gastrointestinal stromal tumor & Imatinib \\
\hline Metastatic hepatocellular carcinoma & Sorafenib \\
\hline Metastatic prostate cancer & Abiraterone, enzalutamide \\
\hline Metastatic renal cell carcinoma & Everolimus, pazopanib, sorafenib, sunitinib \\
\hline Thyroid cancer & Sorafenib
\end{tabular}

no changes to concomitant medication therapies, and no new or worsening medication-related AEs. Patients meeting all criteria were given a 30-day supply of OAN. Prescribing, dispensing, and delivery of OAN were facilitated by the pharmacist. Patient cases that did not meet criteria for renewal were escalated to the hematology/oncology provider or oncology clinical pharmacy specialist for further evaluation.

\section{Study Design and Setting}

This was a pre/post retrospective cohort, quality improvement study of patients enrolled in the RLRVAMC OAN pharmacist renewal clinic. The study was deemed exempt from institutional review board (IRB) by the US Department of Veterans Affairs (VA) Research and Development Department.

\section{Study Population}

Patients were included in the preimplementation group if they had received at least 2 prescriptions of an eligible OAN. Therapy for the preimplementation group was required to be a monthly duration $>21$ days and between the dates of September 1, 2013 and August 31, 2014. Patients were included in the postimplementation group if they had received at least 2 prescriptions of the studied OANs between September 1, 2014 and January 31, 2015. Patients were excluded if they had filled $<2$ prescriptions of OAN; were managed by a non-VA oncologist or hematologist; or received an OAN other than those listed in Table 1.

\section{Data Collection}

For all patients in both the pre- and postimplementation cohorts, a standardized data 
TABLE 2 Adherence and Tolerability Questions asked Within 1 Week of Oral Antineoplastic Renewals

\begin{tabular}{ll} 
No. & Questions \\
\hline $1 \quad \begin{array}{l}\text { People sometimes miss taking their medicines for reasons other than } \\
\text { forgetting. Thinking over the past } 2 \text { weeks, how many doses of your } \\
\text { chemotherapy medicine would you estimate you missed? }\end{array}$ \\
\hline $2 \quad \begin{array}{l}\text { Are you experiencing any new or worsening symptoms that are not being } \\
\text { managed with your current prescriptions? }\end{array}$ \\
\hline $3 \quad \begin{array}{l}\text { What other medicines, including over the counter and herbal } \\
\text { supplements, have you stopped or started taking since you last saw } \\
\text { your doctor? }\end{array}$ \\
\hline $4 \quad \begin{array}{l}\text { How many doses of your chemotherapy medicine would you estimate } \\
\text { you have left? }\end{array}$ \\
\hline $5 \quad \begin{array}{l}\text { Your next appointment with your hematology/oncology provider is on } \\
\text { [date]. Are there any reasons you feel you would need to see them before } \\
\text { that date? }\end{array}$
\end{tabular}

collection tool was used to collect the following via electronic health record review by a PGY2 oncology resident: age, race, gender, oral antineoplastic agent, refill dates, days' supply, estimated unit cost per dose cancer diagnosis, distance from the RLRVAMC, copay status, presence of hospitalizations/ ED visits/dosage reductions, discontinuation rates, reasons for discontinuation, and total number of current prescriptions. The presence or absence of dosage reductions were collected to identify concerns for tolerability, but only the original dose for the preimplementation group and dosage at time of clinic enrollment for the postimplementation group was included in the analysis.

\section{Outcomes and Statistical Analyses}

The primary outcome was medication adherence defined as the median medication possession ratio (MPR) before and after implementation of the clinic. Secondary outcomes included the proportion of patients who were adherent from before implementation to after implementation and estimated cost-savings of this clinic after implementation. MPR was used to estimate medication adherence by taking the cumulative day supply of medication on hand divided by the number of days on therapy. ${ }^{12}$ Number of days on therapy was determined by taking the difference on the start date of the new medication regimen and the discontinuation date of the same regimen. Patients were grouped by adherence into one of the following categories: $<0.8,0.8$ to $0.89,0.9$ to 1 , and $>1.1$.
Patients were considered adherent if they reported taking $\geq 90 \%$ (MPR $\geq 0.9$ ) of prescribed doses, adopted from the study by Anderson and colleagues. ${ }^{12} \mathrm{~A}$ patient with an MPR > 1, likely due to filling prior to the anticipated refill date, was considered $100 \%$ adherent $(M P R=1)$. If a patient switched OAN during the study, both agents were included as separate entities.

A conservative estimate of cost-savings was made by multiplying the RLRVAMC cost per unit of medication at time of initial prescription fill by the number of units taken each day multiplied by the total days' supply on hand at time of therapy discontinuation. Patients with an MPR $<1$ at time of therapy discontinuation were assumed to have zero remaining units on hand and zero cost savings was estimated. Waste, for purposes of cost-savings, was calculated for all MPR values $>1$. Additional supply anticipated to be on hand from dose reductions was not included in the estimated cost of unused medication.

Descriptive statistics compared demographic characteristics between the pre- and postimplementation groups. MPR data were not normally distributed, which required the use of nonparametric Mann-Whitney $U$ tests to compare pre- and postMPRs. Pearson $\chi^{2}$ compared the proportion of adherent patients between groups while descriptive statistics were used to estimate cost savings. Significance was determined based on a $P$ value $<.05$. IBM SPSS Statistics software was used for all statistical analyses. As this was a complete sample of all eligible subjects, no sample size calculation was performed.

\section{Results}

In the preimplementation period, $246 \mathrm{pa}-$ tients received an OAN and 61 patients received an OAN in the postimplementation period (Figure 1). Of the 246 patients in the preimplementation period, 98 were eligible and included in the preimplementation group. Similarly, of the 61 patients in the postimplementation period, 35 patients met inclusion criteria for the postimplementation group. The study population was predominantly male with an average age of approximately 70 years in both groups (Table 3). More than $70 \%$ of the population in each group was White. No statistically significant 
TABLE 3 Patient Demographics

\begin{tabular}{|c|c|c|c|}
\hline Characteristics & $\begin{array}{l}\text { Preimplementation } \\
\qquad(\mathrm{n}=98)\end{array}$ & $\begin{array}{l}\text { Postimplementation } \\
\qquad(\mathrm{n}=35)\end{array}$ & $P$ Value \\
\hline Age, mean (SD), y & $69.1(11.2\}$ & $68.4(12.2)$ & .55 \\
\hline Gender, male, \% & 96 & 100 & .23 \\
\hline Race, White, \% & 79.6 & 71.4 & .46 \\
\hline Copay, \% & 57.1 & 65.7 & .79 \\
\hline Distance from clinic, \% & & & .38 \\
\hline$<30$ miles & 36.7 & 45.8 & -- \\
\hline $31-60$ miles & 23.5 & 25.7 & -- \\
\hline$>60$ miles & 39.8 & 28.5 & -- \\
\hline No. of other medications, mean (SD) & $7.2(4.3)$ & $8.9(4.9)$ & .40 \\
\hline \multicolumn{4}{|l|}{ Oral antineoplastic agent, №. (\%) } \\
\hline Abiraterone & $31(31.6)$ & $9(25.7)$ & -- \\
\hline Dasatinib & $9(9.2)$ & 5 (14.3) & -- \\
\hline Enzalutamide & $12(12.2)$ & $3(8.6)$ & -- \\
\hline Everolimus & $2(2.0)$ & $0(0)$ & -- \\
\hline Imatinib & $23(23.5)$ & $7(20.0)$ & -- \\
\hline Nilotinib & $3(3.1)$ & $2(5.7)$ & -- \\
\hline Pazopanib & $9(9.2)$ & 5 (14.3) & -- \\
\hline Sorafenib & $7(7.1)$ & $2(5.7)$ & -- \\
\hline Sunitinib & $2(2.0)$ & $2(5.7)$ & -- \\
\hline
\end{tabular}

differences between groups were identified. The most commonly prescribed OAN in the preimplementation group were abiraterone, imatinib, and enzalutamide (Table 3). In the postimplementation group, the most commonly prescribed agents were abiraterone, imatinib, pazopanib, and dasatinib. No significant differences were observed in prescribing of individual agents between the pre- and postimplementation groups or other characteristics that may affect adherence including patient copay status, number of concomitant medications, and driving distance from the RLRVAMC.

Thirty-six (36.7\%) patients in the preimplementation group were considered nonadherent $(\mathrm{MPR}<0.9)$ and $18(18.4 \%)$ had an MPR $<0.8$. Fifteen (15.3\%) patients in the preimplementation clinic were considered overadherent (MPR > 1.1). Forty-seven (47.9\%) patients in the preimplementation group were considered adherent (MPR 0.9 - 1.1) while all 35 (100\%) patients in the postimplementation group were considered adherent (MPR 0.9 - 1.1). No non- or overadherent patients were identified in the postimplementation group (Figure 2). The median MPR for all patients in the preimplementation group was
FIGURE 1 Study Cohort Flow Diagram

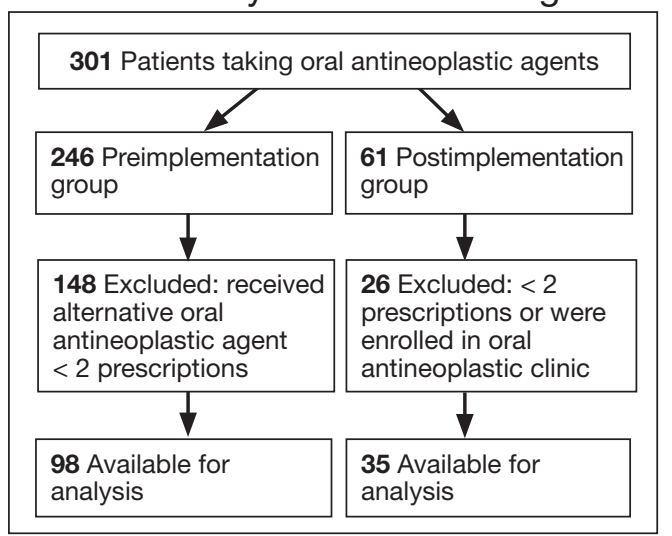

0.94 compared with $1.06(P<.001)$ in the postimplementation group.

Thirty-five $(35.7 \%)$ patients had therapy discontinued or held in the preimplementation group compared with 2 (5.7\%) patients in the postimplementation group $(P<.001)$. Reasons for discontinuation in the preimplementation group included disease progression $(n=27)$, death $(n=3)$, lost to follow up $(\mathrm{n}=2)$, and intolerability of therapy $(n=3)$. Both patients that discontinued therapy in the postimplementation group did so due to disease progression. Of the 35 patients who had their OAN 
discontinued or held in the preimplementation group, 14 patients had excess supply on hand at time of discontinuation. The estimated value of the unused medication was $\$ 37,890$. Nine (25\%) of the 35 patients who discontinued therapy had a dosage reduction during the course of therapy and the additional supply was not included in the cost estimate. Similarly, 1 of the 2 patients in the postimplementation group had their OAN discontinued during study. The cost of oversupply of medication at the time of therapy discontinuation was estimated at $\$ 1,555$. No patients in the postimplementation group had dose reductions. After implementation of the OAN renewal clinic, the total cost savings between pre $(\$ 37,890)$ and postimplementation $(\$ 1,555)$ groups was $\$ 36,355$.

\section{DISCUSSION}

OANs are widely used therapies, with more than 25 million doses administered per year in the United States alone. ${ }^{12}$ The use of these agents will continue to grow as more targeted agents become available and patients request more convenient treatment options. The role for hematology/oncology clinical pharmacy services must adapt to this increased usage of OANs, including increasing pharmacist involvement in medication education, adherence and tolerability assessments, and proactive drug interaction monitoring. However, additional research is needed to determine optimal management strategies.

Our study aimed to compare OAN adherence among patients at a tertiary care VA hospital before and after implementation of a renewal clinic. The preimplementation population had a median MPR of 0.94 compared with 1.06 in the postimplementation group $(P<.001)$. Although an ideal MPR is 1.0 , we aimed for a slightly higher MPR to allow a supply buffer in the event of prescription delivery delays, as more than $90 \%$ of prescriptions are mailed to patients from a regional mail-order pharmacy. Importantly, the median MPRs do not adequately convey the impact from this clinic. The proportion of patients who were considered adherent to OANs increased from $47.9 \%$ in the preimplementation to $100 \%$ in the postimplementation period. These finding suggest that the clinical pharmacist role to assess and encourage adherence through monitoring tolerability of these OANs improved the overall medication taking experience of these patients.

Upon initial evaluation of adherence pre- and postimplementation, median adherence rates in both groups appeared to be above goal at 0.94 and 1.06 respectively. Patients in the postimplementation group intentionally received a 5- to 7-day supply buffer to account for potential prescription delivery delays due to holidays and inclement weather. This would indicate that the patients in the postimplementation group would have $15 \%$ oversupply due to the 5-day supply buffer. After correcting for patients with confounding reasons for excess (dose reductions, breaks in treatment, etc.), the median MPR in the prerefill clinic group decreased to 0.9 and the MPR in the postrefill clinic group increased slightly to 1.08. Although the median adherence rate in both the pre- and postimplementation groups were above goal of $0.90,36 \%$ of the patients in the preimplementation group were considered nonadherent (MPR < 0.9) compared with no patients in the postimplementation group. Therefore, our intervention to improve patient adherence appeared to be beneficial at our institution.

In addition to improving adherence, one of the goals of the renewal clinic was to minimize excess supply at the time of therapy discontinuation. This was accomplished by aligning medication fills with medical visits and objective monitoring, as well as limiting supply to no more than 30 days. Of the patients in the postimplementation group, only 1 patient had remaining medication at the time of therapy discontinuation compared with 14 patients in the preimplementation group. The estimated cost savings from excess supply was $\$ 36,335$. Limiting the amount of unused supply not only saves money for the patient and the institution, but also decreases opportunity for improper hazardous waste disposal and unnecessary exposure of hazardous materials to others.

Our results show the pharmacist intervention in the coordination of renewals improved adherence, minimized medication waste, and saved money. The cost of pharmacist time participating in the refill clinic 
was not calculated. Each visit was completed in approximately 5 minutes, with subsequent documentation and coordination taking an additional 5 to 10 minutes. During the launch of this service, the oncology pharmacy resident provided all coverage of the clinic. Oversite of the resident was provided by hematology/oncology clinical pharmacy specialists. We have continued to utilize pharmacy resident coverage since that time to meet education needs and keep the estimated cost per visit low. Another option in the case that pharmacy residents are not available would be utilization of a pharmacy technician, intern, or professional student to conduct the adherence and tolerability phone assessments. Our escalation protocol allows intervention by clinical pharmacy specialist and/or other health care providers when necessary. Trainees have only required basic training on how to use the protocol.

\section{Limitations}

Due to this study's retrospective design, an inherent limitation is dependence on prescriber and refill records for documentation of initiation and discontinuation dates. Therefore, only the association of impact of pharmacist intervention on medication adherence can be determined as opposed to causation. We did not take into account discrepancies in day supply secondary to 'held' therapies, dose reductions, or doses supplied during an inpatient admission, which may alter estimates of MPR and cost-savings data. Patients in the postimplementation group intentionally received a 5 to 7 -day supply buffer to account for potential prescription delivery delays due to holidays and inclement weather. This would indicate that the patients in the postimplementation group would have $15 \%$ oversupply due to the 5-day supply buffer, thereby skewing MPR values. This study did not account for cost avoidance resulting from early identification and management of toxicity. Finally, the postimplementation data only spans 4 months and a longer duration of time is needed to more accurately determine sustainability of renewal clinic interventions and provide comprehensive evaluation of cost-avoidance.
FIGURE 2 Oral Antineoplastic Medication Adherence

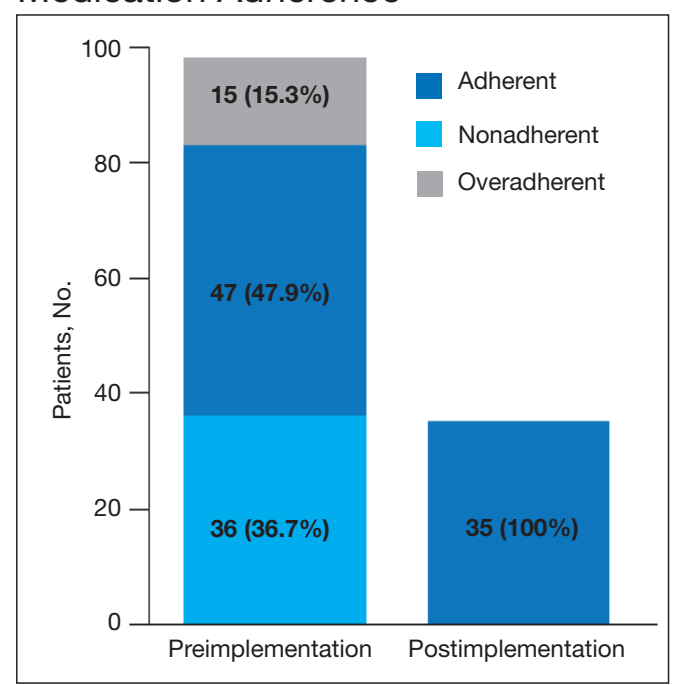

\section{CONCLUSION}

Implementation of an OAN renewal clinic was associated with an increase in MPR, improved proportion of patients considered adherent, and an estimated $\$ 36,335$ cost-savings. However, prospective evaluation and a longer study duration are needed to determine causality of improved adherence and cost-savings associated with a pharmacistdriven OAN renewal clinic.

\section{Author affiliations}

Brooke Crawford and Susan Bullington are Clinical Pharmacy Specialists Hematology/Oncology at the Richard L. Roudebush Veterans Affairs Medical Center in Indianapolis, Indiana. Alison Stauder is a Clinical Pharmacy Specialist Hematology/Oncology at the John Cochran Veterans Affairs Medical Center in St. Louis, Missouri. Patrick Kiel is a Clinical Pharmacy Specialist Precision Genomics at the Indiana University Simon Cancer Center in Indianapolis. Erin Dark is Pharmacy Student at Butler University College of Pharmacy in Lafayette, Indiana. Jill Johnson is a Clinical Hematology/Oncology Pharmacist at in the Minneapolis Veterans Affairs Medical Center in Minneapolis, Minnesota. Alan Zillich is the William S. Bucke Professor and Head of the Purdue University College of Pharmacy Department of Pharmacy Practice in West Lafayette, Indiana.

\section{Author disclosures}

The authors report no actual or potential conflicts of interest with regard to this article.

\section{Disclaimer}

The opinions expressed herein are those of the authors and do not necessarily reflect those of Federal Practitioner, Frontline Medical Communications Inc., the US Government, or any of its agencies. This article may discuss unlabeled or investigational use of certain drugs. Please review the complete prescribing information for specific drugs or drug combinations-including indications, contraindications, warnings, 
and adverse effects - before administering pharmacologic therapy to patients.

\section{References}

1. Ganesan P, Sagar TG, Dubashi B, et al. Nonadherence to imatinib adversely affects event free survival in chronic phase chronic myeloid leukemia. Am J Hematol 2011; 86: 471-474. doi:10.1002/ajh.22019

2. Marin D, Bazeos A, Mahon FX, et al. Adherence is the critical factor for achieving molecular responses in patients with chronic myeloid leukemia who achieve complete cytogenetic responses on imatinib. J Clin Oncol 2010; 28: 2381-2388. doi:10.1200/JCO.2009.26.3087

3. McCowan C, Shearer J, Donnan PT, et al. Cohort study examining tamoxifen adherence and its relationship to mortality in women with breast cancer. Br J Cancer 2008; 99: 1763-1768. doi:10.1038/sj.bjc.6604758

4. Lexicomp Online. Sunitinib. Hudson, Ohio: Lexi-Comp, Inc; August 20, 2019.

5. Babiker A, El Husseini M, Al Nemri A, et al. Health care professional development: Working as a team to improve patient care. Sudan J Paediatr. 2014;14(2):9-16.

6. Spence MM, Makarem AF, Reyes SL, et al. Evaluation of an outpatient pharmacy clinical services program on adherence and clinical outcomes among patients with diabetes and/or coronary artery disease. J Manag Care Spec Pharm. 2014;20(10):1036-1045. doi:10.18553/ jmcp.2014.20.10.1036

7. Holle LM, Puri S, Clement JM. Physician-pharmacist collaboration for oral chemotherapy monitoring: Insights from an academic genitourinary oncology practice. $J$ Oncol Pharm Pract 2015; doi:10.1177/1078155215581524

8. Muluneh B, Schneider M, Faso A, et al. Improved Adherence Rates and Clinical Outcomes of an Integrated, Closed-Loop, Pharmacist-Led Oral Chemotherapy Management Program. Journal of Oncology Practice. 2018;14(6):371-333. doi:10.1200/JOP.17.00039.

9. Font R, Espinas JA, Gil-Gil M, et al. Prescription refill, patient self-report and physician report in assessing adherence to oral endocrine therapy in early breast cancer patients: a retrospective cohort study in Catalonia, Spain. British Journal of Cancer. $2012 ; 107(8): 1249-1256$. doi:10.1038/bjc.2012.389.

10. Anderson KR, Chambers CR, Lam N, et al. Medication adherence among adults prescribed imatinib, dasatinib, or nilotinib for the treatment of chronic myeloid leukemia. J Oncol Pharm Practice. 2015;21(1):19-25. doi:10.1177/1078155213520261

11. Weingart SN, Brown E, Bach PB, et al. NCCN Task Force Report: oral chemotherapy. J Natl Compr Canc Netw. 2008;6(3): S1-S14 